\title{
The fertilization ability and developmental competence of bovine oocytes grown in vitro
}

\author{
Miho MAKITA ${ }^{1)}$, Mayuko UEDA ${ }^{1)}$ and Takashi MIYANO ${ }^{1)}$ \\ 1) Graduate School of Agricultural Science, Kobe University, Kobe 657-8501, Japan
}

\begin{abstract}
In vitro growth culture systems for oocytes are being developed in several mammalian species. In these growth culture systems, in vitro grown oocytes usually have lower blastocyst formation than in vivo grown oocytes after in vitro fertilization. Furthermore, there have been a few reports that investigated the fertilization ability of in vitro grown oocytes in large animals. The purpose of this study was to investigate the fertilization process and developmental competence of bovine oocytes grown in vitro. Oocyte-granulosa cell complexes collected from bovine early antral follicles $(0.4-0.7 \mathrm{~mm}$ in diameter $)$ were cultured for growth with $17 \beta$-estradiol and androstenedione for 14 days and matured in vitro. These oocytes were then inseminated for 6 or $12 \mathrm{~h}$, and further cultured for development up to 8 days in vitro. After growth culture, oocytes grew from $95 \mu \mathrm{m}$ to around $120 \mu \mathrm{m}$ and acquired maturation competence (79\%). Although fertilization rates of in vitro grown oocytes were low after $6 \mathrm{~h}$ of insemination, $34 \%$ of in vitro grown oocytes fertilized normally after $12 \mathrm{~h}$ of insemination, having two polar bodies and two pronuclei with a sperm tail, and $22 \%$ of these oocytes developed into blastocysts after 8 days of culture. The fertilization and blastocyst formation rates were similar to those of in vivo grown oocytes. In addition, blastocyst cell numbers were also similar between in vitro and in vivo grown oocytes. In conclusion, in vitro grown bovine oocytes are similar to in vivo grown oocytes in fertilization ability and can develop into blastocysts.
\end{abstract}

Key words: Cow, In vitro fertilization, Oocyte growth, Oocyte maturation

(J. Reprod. Dev. 62: 379-384, 2016)

$\mathbf{I}_{\mathrm{n}}^{\mathrm{n}}$

order to utilize the huge number of oocytes stored in mammalian

varies, many technologies have been developed. Mammalian oocytes were fertilized in vitro for the first time in the late $1950 \mathrm{~s}$ [1], and successful in vitro fertilizations in several mammals were reported in the 1960s and 1970s. Bovine oocyte in vitro fertilization was first reported in 1977 [2]; subsequently, the first offspring was produced from in vitro fertilized bovine oocytes [3]. These days, in vitro fertilization is a well-established tool and widely used in agricultural and medical fields [4]. More recently, many efforts have been made to develop in vitro growth culture systems for oocytes in a variety of species $[5,6]$. Bovine live offspring have been produced from early antral follicle-derived oocytes after in vitro growth, maturation, and fertilization [7-9].

The in vivo or in vitro environment in which oocytes grow and mature is a major determinant factor of oocyte quality that represents fertilization ability and developmental competence [10, 11]. Although our previous reports $[12,13]$ showed that the culture system using steroid hormones supported bovine oocyte growth and promoted the acquisition of meiotic competence in vitro, oocyte growth culture systems still have a low yield of competent oocytes; thus, there have been a few reports that investigated the fertilization ability of in vitro grown oocytes in large animals. In addition, most of the

Received: January 3, 2016

Accepted: April 6, 2016

Published online in J-STAGE: May 2, 2016

(C)2016 by the Society for Reproduction and Development

Correspondence: M Makita (e-mail: 124a206a@stu.kobe-u.ac.jp)

This is an open-access article distributed under the terms of the Creative Commons Attribution Non-Commercial No Derivatives (by-nc-nd) License

$<$ http://creativecommons.org/licenses/by-nc-nd/4.0/>. reports have evaluated the fertilization ability of in vitro grown oocytes by observing cleavage and blastocyst formation after in vitro fertilization. Nevertheless, the fertilization process of in vitro grown oocytes remains unclear. In this study, we investigated the fertilization of in vitro grown bovine oocytes based on formation of pronuclei, second polar body extrusion, and sperm entry. The oocytes from bovine early antral follicles were cultured for growth, matured, and inseminated in vitro. The fertilization ability and subsequent developmental competence of in vitro grown oocytes were examined.

\section{Materials and Methods}

\section{Chemicals}

All chemicals were purchased from Sigma-Aldrich (St. Louis, MO, USA) unless otherwise stated.

\section{Collection of oocyte-granulosa cell complexes (OGCs)}

Bovine ovaries from Japanese Black cattle were obtained from a local abattoir and transported to the laboratory. The ovaries were washed once in $0.2 \%(\mathrm{wt} / \mathrm{vol})$ cetyltrimethylammonium bromide and three times in Dulbecco's phosphate-buffered saline (PBS) containing $0.1 \%$ (wt/vol) polyvinyl alcohol (PVA) (PBS-PVA). OGCs consisting of oocytes and cumulus/granulosa cells were collected from antral follicles at two different sizes. For the collection of OGCs with fully grown oocytes, follicular fluids containing OGCs were obtained from antral follicles (4-6 $\mathrm{mm}$ in diameter) using needles (18 gauge; Terumo, Tokyo, Japan) and syringes; these OGCs served as the controls. For the collection of OGCs with growing oocytes, ovarian cortical slices (1-1.5 mm) were made using a surgical blade (No. 10; Feather Safety Razor, Tokyo, Japan) and forceps. Antral 
follicles (0.4-0.7 $\mathrm{mm}$ in diameter) were dissected from the cortices under a stereomicroscope (Leica MZ125; Leica Microsystems, Wetzlar, Germany). The follicles were opened with forceps and a blade (No. 10) to isolate OGCs in 25 mM HEPES-buffered medium 199 (HEPES-199; Nissui Pharmaceutical, Tokyo, Japan) containing $0.1 \%$ (wt/vol) PVA, $0.85 \mathrm{mg} / \mathrm{ml}$ sodium bicarbonate (Wako Pure Chemical Industries, Osaka, Japan), and $0.08 \mathrm{mg} / \mathrm{ml}$ kanamycin sulfate. After measuring oocyte diameter (excluding the zona pellucida) to the nearest $1 \mu \mathrm{m}$ using an ocular micrometer attached to an inverted microscope, OGCs that contained oocytes of 90-100 $\mu \mathrm{m}$ in diameter were used for in vitro growth culture.

\section{In vitro growth culture of oocytes}

In vitro growth culture was performed according to the procedure described previously $[8,12]$ with modifications. OGCs were isolated from bovine antral follicles $(0.4-0.7 \mathrm{~mm}$ in diameter) and the day of OGCs isolation was designated day 0. Groups of 10-20 OGCs were cultured in vitro for 14 days on Millicell inserts $(30 \mathrm{~mm}$ diameter, 0.4 $\mu \mathrm{m}$ pore size; Cell Culture Inserts, Merck Millipore, Billerica, MA, USA) placed in Petri dishes (Falcon No. 351008, Becton Dickinson and Co., Bedford, MA, USA) at $38.5^{\circ} \mathrm{C}$ in a controlled atmosphere $\left(5 \% \mathrm{O}_{2}, 5 \% \mathrm{CO}_{2}, 90 \% \mathrm{~N}_{2}\right)$ from day 0 to day 6 , followed by an atmosphere of $5 \% \mathrm{CO}_{2}$ in air from day 7 to day 14 . We employed Millicell inserts in the present study to culture multiple OGCs at the same time and to reduce the time required for medium change, resulting in less contamination risk. In total, $2 \mathrm{ml}$ of medium was placed in the dishes: $1 \mathrm{ml}$ on the membrane and another $1 \mathrm{ml}$ under the membrane. The culture medium for oocyte growth was Alpha Minimum Essential Medium ( $\alpha$-MEM; GIBCO, Invitrogen, Scotland, UK) supplemented with 5\% (vol/vol) fetal bovine serum (FBS; ICN Biomedicals, Costa Mesa, CA, USA), 4\% (wt/vol) polyvinylpyrrolidone (molecular weight 360,000), $4 \mathrm{mM}$ hypoxanthine, $50 \mu \mathrm{g} / \mathrm{ml}$ ascorbic acid 2-glucoside (Hayashibara Biochemical Laboratories, Okayama, Japan), $55 \mu \mathrm{g} / \mathrm{ml}$ cysteine, $0.05 \mu \mathrm{M}$ dexamethasone, 1 $\mathrm{mM}$ sodium pyruvate, $2.2 \mathrm{mg} / \mathrm{ml}$ sodium bicarbonate and $0.08 \mathrm{mg} /$ $\mathrm{ml}$ kanamycin sulfate [8]. Based on our previous report [12], the medium was also supplemented with $10 \mathrm{ng} / \mathrm{ml} 17 \beta$-estradiol and 10 $\mathrm{ng} / \mathrm{ml}$ androstenedione (Tokyo Chemical Industry, Tokyo, Japan). One-half volume of the culture medium was changed with fresh medium every other day after day 4. On day 7 and day 14, OGCs whose structures had collapsed-for example, those that exhibited complete detachment of granulosa cells from oocytes and/or contained oocytes that showed cytoplasmic degeneration-were classified as degenerative OGCs. After 14 days of culture, the diameters of oocytes that were enclosed by granulosa cells and showed no sign of degeneration were measured as described above and subjected to further experiments.

After in vitro growth culture, oocytes were denuded, fixed with acetic acid-ethanol (1:3), and stained with 1\% (wt/vol) aceto-orcein to assess the stage of meiotic division with Nomarski interference microscopy. The oocytes were classified by morphology of the chromatin and nuclear envelope according to the classifications of Motlik et al. [14] and Hirao et al. [15]. The stages for oocytes with an intact germinal vesicle were classified as filamentous chromatin (FC), stringy chromatin (SC), and germinal vesicle I-IV (GV). After germinal vesicle breakdown, stages were classified as metaphase I
(MI), anaphase I and telophase I (AI-TI), and metaphase II (MII).

\section{In vitro maturation of oocytes}

OGCs with growing oocytes were collected from $0.4-0.7 \mathrm{~mm}$ antral follicles and those with surviving oocytes after 14 days of in vitro growth culture were subjected to maturation culture. OGCs with fully grown oocytes collected from 4-6 mm antral follicles were also cultured for maturation as in vivo controls. The OGCs were cultured in $50 \mu \mathrm{l}$ microdrops of the maturation medium covered with paraffin oil at $38.5^{\circ} \mathrm{C}$ under an atmosphere of $5 \% \mathrm{CO}_{2}$ in humidified air for $22 \mathrm{~h}$. Each microdrop contained 4-5 OGCs. The maturation medium was bicarbonate-buffered medium 199 supplemented with $10 \% \mathrm{FBS}, 0.1 \mathrm{mg} / \mathrm{ml}$ sodium pyruvate, $0.1 \mathrm{IU} / \mathrm{ml}$ human menopausal gonadotropin (hMG; Aska Pharmaceutical, Tokyo, Japan), $0.08 \mathrm{mg} /$ $\mathrm{ml}$ kanamycin sulfate, and $2.2 \mathrm{mg} / \mathrm{ml}$ sodium bicarbonate. After culture, OGCs were subjected to subsequent in vitro fertilization. Selected oocytes were denuded mechanically using a small-bore pipette with the help of $0.1 \%$ (wt/vol) hyaluronidase (from bovine testes), then fixed and stained to assess the stage of meiotic division as described above.

\section{In vitro fertilization and development}

For in vitro fertilization, straws with frozen semen (Japanese Black, P706, Livestock Improvement Association of Japan, Tokyo, Japan) were thawed in a water bath at $37^{\circ} \mathrm{C}$. The spermatozoa were washed twice by centrifugation. First, they were washed $(760 \times g)$ for $20 \mathrm{~min}$ over a two-step gradient with $2 \mathrm{ml}$ of $90 \%$ and $2 \mathrm{ml}$ of $45 \%$ Percoll (GE Healthcare UK, Buckinghamshire, England, UK) in PBS, then washed $(372 \times g)$ in $3 \mathrm{ml}$ of insemination medium (IVF100, Research Institute for the Functional Peptides, Yamagata, Japan) for $5 \mathrm{~min}$. The final concentration of spermatozoa was adjusted to $2.8-4.8 \times 10^{6}$ sperm $/ \mathrm{ml}$ by dilution with the insemination medium. Depending on the available number of OGCs, they were then transferred to 50 or $100 \mu 1$ microdrops of sperm suspension (50 and $100 \mu 1$ microdrops contained 10-13 and 20-25 OGCs, respectively) and incubated for 6 or $12 \mathrm{~h}$ at $38.5^{\circ} \mathrm{C}$ in a $5 \% \mathrm{CO}_{2}$ humidified atmosphere. After insemination, some OGCs were subjected to evaluation of fertilization, while others were subjected to evaluation of development. In instances when the number of OGCs were insufficient for both experiments, they were subjected to either of the two experiments.

For evaluation of fertilization, the mean $( \pm$ SEM) concentrations of spermatozoa in insemination medium for in vivo and in vitro grown OGCs were $3.4 \pm 0.1 \times 10^{6} \mathrm{sperm} / \mathrm{ml}(\mathrm{n}=3)$ and $3.3 \pm 0.3 \times$ $10^{6} \mathrm{sperm} / \mathrm{ml}(\mathrm{n}=4)$, respectively. For evaluation of development, the mean $( \pm$ SEM) concentrations of spermatozoa for in vivo and in vitro grown OGCs were $3.3 \pm 0.2 \times 10^{6} \mathrm{sperm} / \mathrm{ml}(\mathrm{n}=6)$ and $3.5 \pm$ $0.3 \times 10^{6} \mathrm{sperm} / \mathrm{ml}(\mathrm{n}=7)$, respectively. There were no significant differences between the sperm concentrations of in vivo and in vitro grown OGCs (unpaired $t$ test, $\mathrm{P}>0.05$ ).

To assess fertilization, some of the oocytes were denuded using a small-bore pipette after 6 or $12 \mathrm{~h}$ of insemination, followed by in vitro development (10 or $4 \mathrm{~h}$, respectively) for a total of $16 \mathrm{~h}$ after insemination. The oocytes were then fixed with acetic acid-ethanol (1:3) and stained with $1 \%$ (wt/vol) aceto-orcein. Oocytes that had two polar bodies and two pronuclei with a sperm tail were classified as normal fertilization. Fertilization other than normal fertilization-for 
example, oocytes that had an enlarged sperm head with anaphase/ telophase I chromosomes, two pronuclei with a sperm tail and one polar body, or more than two sperm heads or pronuclei-were classified as abnormal fertilization. MI, AI-TI, or MII oocytes and oocytes with one or two pronuclei without sperm tails were classified as unfertilized oocytes. Oocytes that showed cytoplasmic degeneration were classified as degenerated oocytes.

After in vitro fertilization, remaining oocytes were denuded mechanically and subjected to development culture. The day of in vitro fertilization was designated day 0 . Oocytes (presumptive zygotes) were cultured in $50 \mu \mathrm{l}$ microdrops of serum free medium (IVD101, Research Institute for the Functional Peptides) for up to 8 days. Each microdrop contained 5-7 oocytes. The rates of blastocyst formation on day 8 were recorded. After 7 days of development culture, several randomly selected blastocysts from in vivo grown oocytes and in vitro grown oocytes were washed twice in PBS-PVA for $15 \mathrm{~min}$ each and fixed in 4\% (wt/vol) paraformaldehyde in PBS-PVA for 60 min. Fixed blastocysts were washed twice in PBS-PVA for $15 \mathrm{~min}$ each and blocked in PBS-PVA containing $1 \mathrm{mg} / \mathrm{ml}$ bovine serum albumin for $60 \mathrm{~min}$. Blastocysts were then stained with ProLong Gold Antifade Regent with DAPI (P36931; Molecular Probes, Invitrogen, Carlsbad, CA, USA) and observed under a fluorescence microscope (BX53F; Olympus, Tokyo, Japan) for cell counting.

\section{Statistical analysis}

The frequencies of oocytes at each stage of the meiotic division were analyzed using Chi-square test. Differences among the mean $( \pm$ SEM) diameters of oocytes were analyzed using one-way ANOVA followed by the Tukey-Kramer multiple range test (Excel software with the add-in Ekuseru-Toukei 2010; Social Survey Research Information, Tokyo, Japan). For statistical analyses of fertilization rate and blastocyst formation, data were subjected to one-way ANOVA followed by the Tukey-Kramer multiple range test. Differences in cell numbers of blastocysts were analyzed by an unpaired $t$ test. Values of $\mathrm{P}<0.05$ were considered significant.

\section{Results}

\section{In vitro growth and maturation of oocytes}

Figure 1A shows the morphology of OGCs during growth culture. On day 1, each complex attached to the membrane and started to grow, and antrum-like cavities were formed in the granulosa cell
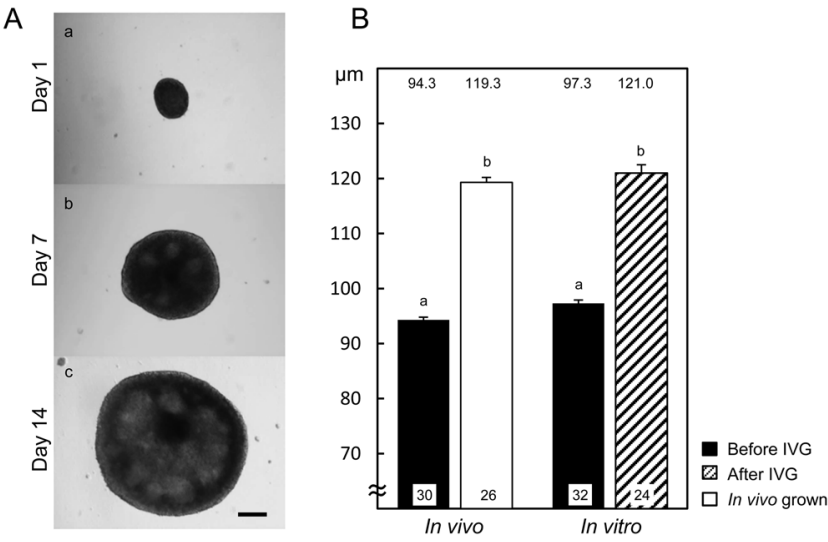

Fig. 1. Typical morphology of bovine OGCs during growth culture (A) and the diameters of oocytes after growth culture (B). (A) OGCs were cultured for 14 days. A scale bar represents $200 \mu \mathrm{m}$. (B) The black bars indicate the diameters of oocytes isolated from 0.4-0.7 mm antral follicles before in vitro growth culture (IVG). The blank bar and striped bars represent the diameters of oocytes collected from 4-6 mm antral follicles (In vivo) and after 14 days of growth culture (In vitro), respectively. The numbers of oocytes examined are shown at the bottom of each bar, and the numbers above the bars indicate the mean diameters of oocytes $(\mu \mathrm{m})$. a, b Values differ significantly $(\mathrm{P}<0.05)$

layers by day 7 . The dome-like structures were further developed during culture. On day $14,75 \%$ of OGCs maintained structures that contained viable oocytes. The mean diameters of oocytes collected from $0.4-0.7 \mathrm{~mm}$ antral follicles were around $95 \mu \mathrm{m}$ (Fig. 1B). After 14 days of growth culture, the mean diameter of oocytes was increased to $121.0 \pm 1.3 \mu \mathrm{m}$, and the diameter was comparable to that of in vivo grown oocytes $(119.3 \pm 1.0 \mu \mathrm{m})$.

Table 1 shows the meiotic competence of in vitro grown oocytes from $0.4-0.7 \mathrm{~mm}$ antral follicles. After $22 \mathrm{~h}$ of maturation culture, all oocytes without growth culture remained at the FC (57\%) or SC (43\%) stage, whereas all oocytes with growth culture resumed meiosis. Both in vivo and in vitro OGCs underwent cumulus expansion during the maturation culture (Fig. 2), and some of the oocytes reached MII after culture; The MII rate of in vitro grown oocytes (79\%) was comparable to that of in vivo grown oocytes $(81 \%)$. When the total number of oocytes initially used for growth culture

Table 1. Meiotic competence of in vitro grown bovine oocytes

\begin{tabular}{|c|c|c|c|c|c|c|c|c|}
\hline \multirow{2}{*}{ In vitro growth (day) ${ }^{1}$} & \multicolumn{2}{|c|}{ No. of oocytes used ${ }^{2}$} & \multicolumn{6}{|c|}{ No. $(\%)$ of oocytes at the stage of ${ }^{3}$} \\
\hline & IVG & IVM & FC & $\mathrm{SC}$ & GV & MI & $\mathrm{AI}-\mathrm{TI}$ & $\mathrm{MII}^{4}$ \\
\hline 0 & - & 30 & $17(57)$ & $13(43)$ & $0(0)$ & $0(0)$ & $0(0)$ & $0(0)$ \\
\hline 14 & 32 & 24 & $0(0)$ & $0(0)$ & $0(0)$ & $2(8)$ & $3(13)$ & 19 (79) [59] \\
\hline In vivo & - & 26 & $0(0)$ & $0(0)$ & $0(0)$ & $5(19)$ & $0(0)$ & $21(81)$ \\
\hline
\end{tabular}

${ }^{1}$ OGCs collected from $0.4-0.7 \mathrm{~mm}$ antral follicles were subjected to in vitro maturation culture before (0) or after 14 days of in vitro growth culture (14). OGCs from 4-6 mm antral follicles were subjected to in vitro maturation culture as in vivo controls (In vivo). ${ }^{2}$ After in vitro growth culture (IVG), OGCs with surviving oocytes were transferred to in vitro maturation culture (IVM). ${ }^{3}$ FC: filamentous chromatin, SC: stringy chromatin, GV: germinal vesicle I-IV, MI: metaphase I, AI-TI: anaphase I and telophase I, MII: metaphase II. ${ }^{4}$ The number in [ ] indicates the percentage of MII oocytes from the oocytes initially used for IVG. 

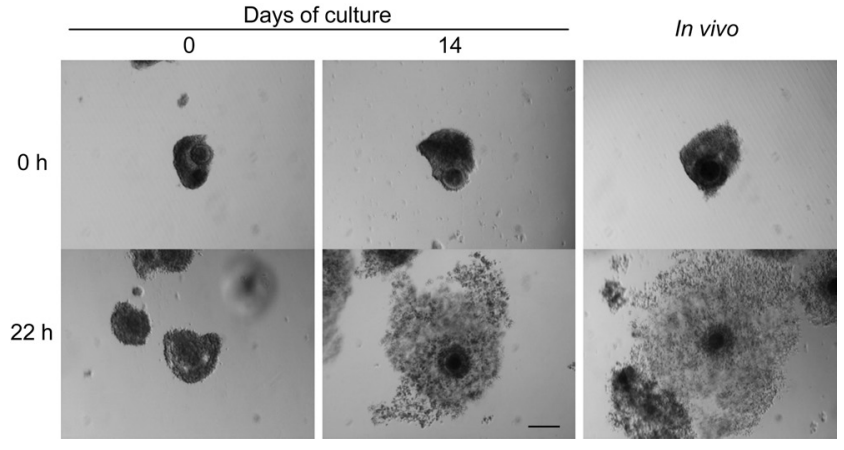

Fig. 2. Representative morphology of bovine OGCs before and after maturation culture. After 14 days of growth culture, OGCs with surviving oocytes were subjected to maturation culture for $22 \mathrm{~h}$. OGCs collected from $0.4-0.7 \mathrm{~mm}$ antral follicles $(0)$ and $4-6 \mathrm{~mm}$ antral follicles (In vivo) were cultured as for in vivo control. A scale bar represents $200 \mu \mathrm{m}$.

was taken into consideration, $59 \%$ of oocytes matured to MII after maturation culture.

\section{Fertilization ability of oocytes}

After maturation culture, oocytes grown in vivo and in vitro were fertilized in vitro to assess their fertilization ability. After 6 and 12 $\mathrm{h}$ of insemination, oocytes grown in vivo were fertilized normally (38 and 37\%, respectively) (Table 2). All of these oocytes had two polar bodies and two pronuclei with a sperm tail (Fig. 3A). There were no significant differences in rates of total unfertilized in vivo grown oocytes between 6 and $12 \mathrm{~h}$ of insemination (52 and $41 \%$, respectively); however, the rate of unfertilized MII oocytes significantly decreased, and the rate of oocytes that formed pronuclei without corresponding sperm tail (classified as Others in Table 2) increased after $12 \mathrm{~h}$ of insemination. Among in vitro grown oocytes, only $3 \%$ of oocytes showed normal fertilization and most of the oocytes $(74 \%)$ were unfertilized after $6 \mathrm{~h}$ of insemination (Table 2 ). After $12 \mathrm{~h}$ of insemination, the rate of unfertilized oocytes significantly decreased, especially for MII oocytes; conversely the normal fertilization rate increased to $34 \%$ and was similar to that of in vivo grown oocytes. Normal fertilized oocytes had two polar bodies and two pronuclei with a sperm tail (Fig. 3B). Abnormal fertilization rates of in vitro grown oocytes were slightly higher than those of in vivo grown oocytes for both 6 and $12 \mathrm{~h}$ of insemination.

\section{Developmental competence of oocytes}

The developmental competences of in vivo and in vitro grown oocytes were assessed by development culture up to 8 days (Table 3 ). After insemination for 6 and $12 \mathrm{~h}$, oocytes grown in vivo developed into blastocysts ( $23 \%$ and $22 \%$, respectively) following development culture for 8 days. Although almost none of the in vitro grown oocytes that were inseminated for $6 \mathrm{~h}$ developed into blastocysts after 8 days of culture ( $1 \%), 22 \%$ of the oocytes developed into blastocysts when they were inseminated for $12 \mathrm{~h}$. This blastocyst formation rate was comparable to that of in vivo grown oocytes.

To assess the quality of blastocysts derived from in vitro grown oocytes, cell numbers of randomly selected blastocysts were counted on day 7 of in vitro development (Fig. 4). The mean numbers of blastocyst cells derived from in vivo and in vitro grown oocytes were $90.5 \pm 4.0(n=9)$ and $90.8 \pm 5.3(n=8)$, respectively; these numbers were comparable with each other.

\section{Discussion}

During oocyte growth phase in the ovary, oocytes increase their volume, acquire maturation competence, and accumulate the materials necessary for prospective fertilization and development. In this study, we showed that in vitro growth culture supported the acquisition of fertilization ability and developmental competence, as well as complete oocyte growth and meiotic competence during in vitro growth.

After in vitro maturation and fertilization, $22 \%$ of in vitro grown oocytes inseminated for $12 \mathrm{~h}$ developed into blastocysts following development culture for 8 days. This blastocyst formation rate was similar to that of in vivo grown oocytes. Since blastocyst cell numbers were also similar between in vitro and in vivo grown oocytes, the quality of blastocysts derived from in vitro grown oocytes appeared to be comparable to that from in vivo grown oocytes. Moreover, more than one third of oocytes grown in vivo and in vitro were fertilized

Table 2. Fertilization ability of in vitro grown bovine oocytes after in vitro maturation

\begin{tabular}{|c|c|c|c|c|c|c|c|c|c|c|c|}
\hline \multirow{3}{*}{ Growth ${ }^{1}$} & \multirow{3}{*}{$\begin{array}{c}\text { Insemination } \\
\text { (h) }\end{array}$} & \multirow{3}{*}{$\begin{array}{c}\text { No. of oocytes } \\
\text { examined } \\
\text { (replicates) }\end{array}$} & \multicolumn{4}{|c|}{ Fertilized oocytes $(\%)^{2}$} & \multirow{2}{*}{\multicolumn{4}{|c|}{ Unfertilized oocytes $(\%)^{3}$}} & \multirow{3}{*}{$\mathrm{DG}^{4}$} \\
\hline & & & \multirow{2}{*}{$\begin{array}{c}\text { Normal } \\
\text { 2PN2PB }\end{array}$} & \multicolumn{3}{|c|}{ Abnormal fertilization } & & & & & \\
\hline & & & & Polyspermy & Others & Total & MI & MII & Others & Total & \\
\hline \multirow[t]{2}{*}{ In vitro } & 6 & $31(4)$ & $1(3)^{\mathrm{a}}$ & $4(13)^{\mathrm{a}}$ & $3(9)^{\mathrm{ab}}$ & $7(23)^{\mathrm{ab}}$ & $3(10)$ & $11(35)^{\mathrm{a}}$ & $9(29)^{\mathrm{ab}}$ & $23(74)^{\mathrm{a}}$ & $0(0)$ \\
\hline & 12 & $32(4)$ & $11(34)^{b}$ & $5(16)^{\mathrm{a}}$ & $6(19)^{\mathrm{b}}$ & $11(34)^{b}$ & $4(13)$ & $2(6)^{b}$ & $4(13)^{\mathrm{a}}$ & $10(31)^{\mathrm{b}}$ & $0(0)$ \\
\hline \multirow[t]{2}{*}{ In vivo } & 6 & $50(3)$ & $19(38)^{b}$ & $1(2)^{b}$ & $2(4)^{\mathrm{a}}$ & $3(6)^{\mathrm{a}}$ & $4(8)$ & $16(32)^{\mathrm{a}}$ & $6(12)^{\mathrm{a}}$ & $26(52)^{\mathrm{ab}}$ & $2(4)$ \\
\hline & 12 & $49(3)$ & $18(37)^{\mathrm{b}}$ & $3(6)^{\mathrm{ab}}$ & $6(12)^{\mathrm{ab}}$ & $9(18)^{\mathrm{ab}}$ & $1(2)$ & $2(4)^{b}$ & $17(35)^{\mathrm{b}}$ & $20(41)^{\mathrm{ab}}$ & $2(4)$ \\
\hline
\end{tabular}

${ }^{1}$ OGCs collected from $0.4-0.7 \mathrm{~mm}$ antral follicles were subjected to in vitro growth culture with $10 \mathrm{ng} / \mathrm{ml} 17 \beta$-estradiol (E $\left.\mathrm{E}_{2}\right)$ and $10 \mathrm{ng} / \mathrm{ml}$ androstenedione $\left(\mathrm{A}_{4}\right)$ for 14 days (In vitro). Examined oocytes from 4-6 mm antral follicles were used as in vivo controls (In vivo). ${ }^{2}$ Normal fertilization: the oocytes that have two polar bodies and two pronuclei with a sperm tail (2PN2PB). Abnormal fertilization: fertilization other than normal fertilization; for example, the oocytes have an enlarged sperm head with anaphase/telophase I chromosomes, two pronuclei with a sperm tail and one polar body, or more than two sperm heads or pronuclei (polyspermy). ${ }^{3}$ Metaphase I (MI), or metaphase II (MII) oocytes and oocytes that have one or two pronuclei without sperm tails (Others) were classified as unfertilized oocytes. ${ }^{4}$ Degenerated oocytes. ${ }^{\mathrm{a}, \mathrm{b}}$ Values with different superscripts in the same column differ significantly $(\mathrm{P}<0.05)$. 

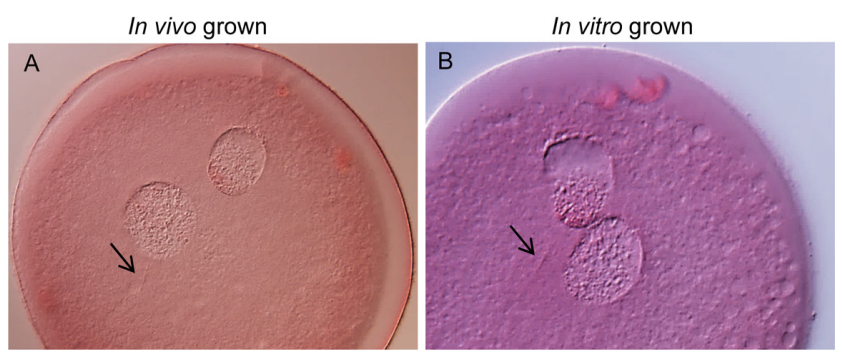

Fig. 3. Morphology of in vivo and in vitro grown bovine oocytes after in vitro fertilization. After $16 \mathrm{~h}$ of insemination, both in vivo (A) and in vitro (B) grown oocytes had two pronuclei and two polar bodies. The arrows indicate sperm tails.

Table 3. Developmental competence of in vitro grown bovine oocytes after in vitro maturation and fertilization

\begin{tabular}{cccc}
\hline Growth $^{1}$ & Insemination (h) & $\begin{array}{c}\text { No. of oocytes used } \\
\text { (replicates) }\end{array}$ & $\begin{array}{c}\text { No. of blastocysts } \\
(\%)^{3}\end{array}$ \\
\hline In vitro & 6 & $95(7)$ & $1(1)^{\mathrm{a}}$ \\
& 12 & $98(7)$ & $22(22)^{\mathrm{b}}$ \\
In vivo & 6 & $136(6)$ & $31(23)^{\mathrm{b}}$ \\
& 12 & $126(6)$ & $28(22)^{\mathrm{b}}$ \\
\hline
\end{tabular}

${ }^{1}$ OGCs collected from $0.4-0.7 \mathrm{~mm}$ antral follicles were subjected to in vitro growth culture for 14 days followed by in vitro maturation and fertilization (In vitro). Examined oocytes from 4-6 $\mathrm{mm}$ antral follicles were used as in vivo controls (In vivo). ${ }^{2}$ Number of oocytes used for in vitro fertilization. ${ }^{3}$ After in vitro fertilization, oocytes were further cultured for development up to 8 days. ${ }^{\text {a, b }}$ Values with different superscripts in the same column differ significantly $(\mathrm{P}<0.05)$.

normally after $16 \mathrm{~h}$ of insemination, having two pronuclei and two polar bodies. These results indicated that in vitro grown oocytes have normal fertilization ability and are competent to develop into blastocysts.

However, few in vitro grown oocytes were fertilized normally after $6 \mathrm{~h}$ of insemination, and almost none of them developed into blastocysts after 8 days of culture. The rate of unfertilized oocytes decreased and normal fertilized in vitro grown oocytes increased to $34 \%$ only after $12 \mathrm{~h}$ of insemination. Meanwhile, there were no significant changes in rates of normal fertilized and total unfertilized in vivo grown oocytes between 6 and $12 \mathrm{~h}$ of insemination. Although the rate of unfertilized MII oocytes decreased, the normal fertilization rate was not increased even after $12 \mathrm{~h}$ of insemination. Unfertilized oocytes seemed to undergo parthenogenetic activation, since they formed pronuclei without corresponding sperm tail. These results suggested that fertilization of in vitro grown oocytes was delayed compared with that of in vivo grown oocytes.

Since spermatozoa have to penetrate cumulus cell layers and the zona pellucida to reach the oocyte plasma membrane, cumulus cells and the zona pellucida are key factors of fertilization. Cumulus expansion during oocyte maturation is essential for normal fertilization [16], and the cumulus cells in expanded cumulus induce sperm capacitation [17]. Since these abilities are considered to be regulated by oocyte
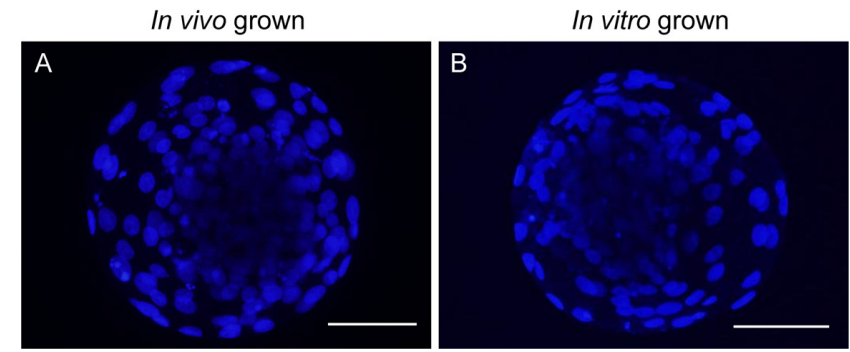

Fig. 4. Bovine blastocysts derived from in vivo and in vitro grown oocytes. Blastocysts (Day 7 of in vitro development) derived from in vivo grown oocytes isolated from 4-6 $\mathrm{mm}$ antral follicles (A) and in vitro grown oocytes growth-cultured with $\mathrm{E}_{2}$ and $\mathrm{A}_{4}(\mathrm{~B})$ were stained with DAPI. Scale bars represent $100 \mu \mathrm{m}$.

secreted paracrine factors during oocyte growth [18], it is suggested that in vitro cultured cumulus cells might not acquire enough ability to expand completely or to induce the sperm capacitation needed for fertilization. After penetration cumulus cell layers, spermatozoa hydrolyze and penetrate the zona pellucida to reach the oocyte plasma membrane. The zona pellucida is formed during oocyte growth and increase in thickness as oocytes increase in diameter [19]. Although zona pellucida glycoproteins are exclusively synthesized by oocytes in mice [20], it is considered that both oocytes and granulosa cells synthesize the zona pellucida in bovine follicles [21, 22]. Longterm growth culture may influence the zona pellucida glycoprotein production in oocytes and/or granulosa cells and thus, influence the morphology or function of the zona pellucida. Although we did not examine the zona pellucida properties of in vitro grown oocytes in this experiment, it is inferred that in vitro grown oocytes took longer to be penetrated by spermatozoa than in vivo grown oocytes due to specific factors (cumulus cells and the zona pellucida). Alternatively, the oocyte itself could also be a factor that influences the fertilization of in vitro grown oocytes. For successful fertilization and subsequent development, both nuclear maturation and cytoplasmic maturation of oocytes are important [23], and oocytes acquire these competences during their growth phase. Since abnormal fertilization rates of in vitro grown oocytes were generally higher than those of in vivo grown oocytes, it is thought that some of the in vitro grown oocytes might not accomplish cytoplasmic maturation during in vitro maturation culture despite of the resulting MII percentage that was similar to that of in vivo grown oocytes.

Oocytes ultimately formed two polar bodies and two pronuclei with a sperm tail at $16 \mathrm{~h}$ of insemination after fertilization and developed normally into blastocysts after 8 days of culture. The cell number of blastocysts was also normal. In conclusion, in vitro grown bovine oocytes are similar to in vivo grown oocytes in fertilization ability and are competent to develop into blastocysts.

\section{Acknowledgments}

We are grateful to the staff of Kobe-Branch, Animal Biotechnology Center, Livestock Improvement Association of Japan, Inc., for supplying ovaries and the staff of Okayama A.I. Center, Livestock Improvement Association of Japan, Inc., for supplying 
frozen semen. This work was supported in part by Japan Society for the Promotion of Science KAKENHI Grant Number 25292192 (to TM).

\section{References}

1. Chang MC. Fertilization of rabbit ova in vitro. Nature 1959; 184(Suppl 7): 466-467. [Medline] [CrossRef]

2. Iritani A, Niwa K. Capacitation of bull spermatozoa and fertilization in vitro of cattle follicular oocytes matured in culture. J Reprod Fertil 1977; 50: 119-121. [Medline] [CrossRef]

3. Brackett BG, Bousquet D, Boice ML, Donawick WJ, Evans JF, Dressel MA. Normal development following in vitro fertilization in the cow. Biol Reprod 1982; 27: 147-158. [Medline] [CrossRef]

4. Bavister BD. Early history of in vitro fertilization. Reproduction 2002; 124: 181-196. [Medline] [CrossRef]

5. Miyano T. In vitro growth of mammalian oocytes. J Reprod Dev 2005; 51: 169-176. [Medline] [CrossRef]

6. Picton HM, Harris SE, Muruvi W, Chambers EL. The in vitro growth and maturation of follicles. Reproduction 2008; 136: 703-715. [Medline] [CrossRef]

7. Yamamoto K, Otoi T, Koyama N, Horikita N, Tachikawa S, Miyano T. Development to live young from bovine small oocytes after growth, maturation and fertilization in vitro. Theriogenology 1999; 52: 81-89. [Medline] [CrossRef]

8. Hirao Y, Itoh T, Shimizu M, Iga K, Aoyagi K, Kobayashi M, Kacchi M, Hoshi H, Takenouchi N. In vitro growth and development of bovine oocyte-granulosa cell complexes on the flat substratum: effects of high polyvinylpyrrolidone concentration in culture medium. Biol Reprod 2004; 70: 83-91. [Medline] [CrossRef]

9. Huang W, Kang SS, Nagai K, Yanagawa Y, Takahashi Y, Nagano M. Mitochondrial activity during pre-maturational culture in in vitro-grown bovine oocytes is related to maturational and developmental competences. Reprod Fertil Dev 2014; 28: 349-356. [Medline] [CrossRef]

10. Lequarre AS, Vigneron C, Ribaucour F, Holm P, Donnay I, Dalbiès-Tran R, Callesen $\mathbf{H}$, Mermillod P. Influence of antral follicle size on oocyte characteristics and embryo development in the bovine. Theriogenology 2005; 63: 841-859. [Medline] [CrossRef]

11. Krisher RL. In vivo and in vitro environmental effects on mammalian oocyte quality. Annu Rev Anim Biosci 2013; 1: 393-417. [Medline] [CrossRef]

12. Makita M, Miyano T. Steroid hormones promote bovine oocyte growth and connection with granulosa cells. Theriogenology 2014; 82: 605-612. [Medline] [CrossRef]

13. Makita M, Miyano T. Androgens promote the acquisition of maturation competence in bovine oocytes. J Reprod Dev 2015; 61: 211-217. [Medline] [CrossRef]

14. Motlík J, Koefoed-Johnsen HH, Fulka J. Breakdown of the germinal vesicle in bovine oocytes cultivated in vitro. J Exp Zool 1978; 205: 377-383. [Medline] [CrossRef]

15. Hirao Y, Tsuji Y, Miyano T, Okano A, Miyake M, Kato S, Moor RM. Association between $\mathrm{p} 34^{\text {cdc2 }}$ levels and meiotic arrest in pig oocytes during early growth. Zygote 1995; 3: 325-332. [Medline] [CrossRef]

16. Chen L, Russell PT, Larsen WJ. Functional significance of cumulus expansion in the mouse: roles for the preovulatory synthesis of hyaluronic acid within the cumulus mass. Mol Reprod Dev 1993; 34: 87-93. [Medline] [CrossRef]

17. Shimada M, Yanai Y, Okazaki T, Noma N, Kawashima I, Mori T, Richards JS Hyaluronan fragments generated by sperm-secreted hyaluronidase stimulate cytokine/ chemokine production via the TLR2 and TLR4 pathway in cumulus cells of ovulated COCs, which may enhance fertilization. Development 2008; 135: 2001-2011. [Medline] [CrossRef]

18. Elvin JA, Clark AT, Wang P, Wolfman NM, Matzuk MM. Paracrine actions of growth differentiation factor-9 in the mammalian ovary. Mol Endocrinol 1999; 13: 1035-1048. [Medline] [CrossRef]

19. Wassarman PM. Zona pellucida glycoproteins. J Biol Chem 2008; 283: 24285-24289. [Medline] [CrossRef]

20. Wassarman PM, Litscher ES. Mammalian fertilization: the egg's multifunctional zona pellucida. Int J Dev Biol 2008; 52: 665-676. [Medline] [CrossRef]

21. Kölle S, Sinowatz F, Boie G, Palma G. Differential expression of ZPC in the bovine ovary, oocyte, and embryo. Mol Reprod Dev 1998; 49: 435-443. [Medline] [CrossRef]

22. Sinowatz F, Kölle S, Töpfer-Petersen E. Biosynthesis and expression of zona pellucida glycoproteins in mammals. Cells Tissues Organs 2001; 168: 24-35. [Medline] [CrossRef]

23. Ferreira EM, Vireque AA, Adona PR, Meirelles FV, Ferriani RA, Navarro PA. Cytoplasmic maturation of bovine oocytes: structural and biochemical modifications and acquisition of developmental competence. Theriogenology 2009; 71: 836-848. [Medline] [CrossRef] 